\title{
Layer-by-Layer Fabrication of Oriented Porous Thin Films Based on Porphyrin-Containing Metal-Organic Frameworks
}

\author{
Monica C. So, ${ }^{\dagger}$ Shengye Jin, ${ }^{\ddagger}$ Ho-Jin Son, ${ }^{\dagger}$ Gary P. Wiederrecht, ${ }^{*}{ }^{\ddagger}$ Omar K. Farha, ${ }^{* \dagger}$ \\ and Joseph T. Hupp* ${ }^{* \dagger}$ \\ ${ }^{\dagger}$ Department of Chemistry, Northwestern University, 2145 Sheridan Road, Evanston, Illinois 60208, United States \\ ${ }^{\ddagger}$ Nanoscience \& Technology Division, Argonne National Laboratory, 9700 South Cass Avenue, Argonne, Illinois 60439, United \\ States
}

Supporting Information

ABSTRACT: We report the synthesis and characterization of two thin films (DA-MOF and L2-MOF) of porphyrin-based MOFs on functionalized surfaces using a layer-by-layer (LbL) approach. Profilometry measurements confirm that the film thickness increases systematically with number of growth cycles. Polarization excitation and fluorescence measurements indicate that the porphyrin units are preferentially oriented, while X-ray reflectivity scans point to periodic ordering. Ellipsometry measurements show that the films are highly porous. Since there are currently few methods capable of yielding microporous MOFs containing accessible free-base porphyrins, it is noteworthy that the LbL growth permits direct MOF incorporation of unmetalated porphyrins. Long-range energy transfer is demonstrated for both MOF films. The findings offer useful insights for subsequent fabrication of MOF-based solar energy conversion devices.

R ecent work on metal-organic frameworks (MOFs) R. (hybrid materials composed of organic linkers and inorganic nodes) has drawn attention to their potential for light harvesting ${ }^{1}$ and for facilitating long-distance energy migration. ${ }^{2-6}$ The crystalline and modular nature of MOFs provides a platform for highly ordered chromophoric structures that mimic those found in natural photosynthesis, such as chlorophylls and carotenoids. ${ }^{7-10}$ A few important studies have previously demonstrated the promise of MOFs for conversion of solar energy to electrical or chemical energy. For example, Lin, Meyer, Papanikolas et al. have reported on the fabrication and energy migration dynamics of metal-bipyridine-derived MOFs for light-harvesting., 311

We have reported the successful synthesis of metalloporphyrin-derived MOFs for light harvesting ${ }^{1}$ and ultrafast long-distance energy migration. ${ }^{4}$ Since metalloporphyrin molecules are structurally similar to light-harvesting and photosynthetic pigments (e.g., chlorins, chlorophylls, etc.), porphyrin-based MOFs might be expected to mimic important aspects of natural photosynthetic systems. The pillared paddlewheel type MOF, DA-MOF, ${ }^{4}$ was synthesized from P1 [5,15-di(4-pyridylacetyl)-10,20-diphyenyl] porphyrinatozinc(II), L1 (1,2,4,5-tetrakis(4-carboxyphenyl)benzene), and zinc(II) acetate $\left(\mathrm{Zn}_{2}{ }^{4+}\right)$ (Figure 1). Our previous studies demonstrated that a photogenerated exciton in a DA-MOF (a)

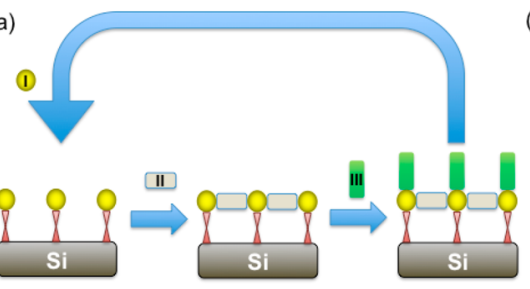

(b)

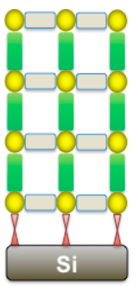

(c) $8=$ 3-APTMS $O=Z_{2}{ }^{4+}$

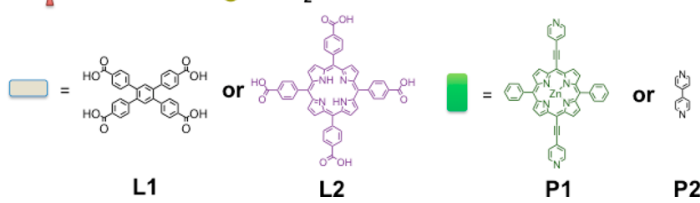

Figure 1. (a) Schematic diagram for the step-by-step growth of the DA-MOF and L2-MOF structures on idealized 3-APTMS, resulting in (b) a film after $N$ cycles of growth. (c) Representations of building blocks used in fabrication of MOF film.

single crystal can accomplish $\sim 2000$ linker-to-linker hops within its brief $(\sim 3 \mathrm{~ns})$ lifetime. ${ }^{4}$ Furthermore, the hopping is directional, resulting in anisotropic energy migration. ${ }^{4}$ The long distance and directional exciton migration suggest that chromophoric MOFs may be superior to monomer dye molecules as light harvesting and energy transport components in solar energy conversion devices.

Unfortunately, how MOF single crystals might be reasonably incorporated into a solar energy conversion device, especially if effective use requires precise optimization of optical path length and crystal-thickness, remains unclear. An attractive alternative would be to directly synthesize MOFs as supported thin films. Several methods ${ }^{12}$ have been developed. ${ }^{13-15}$ Most notable is the step-by-step liquid-phase approach that deposits alternating solutions of structural components onto functionalized substrates (Figure 1). ${ }^{16-18}$ The resulting MOF films are often ultrathin, oriented, and thickness adjustable. The generality and simplicity of the method allow for its exploitation for the preparation of molecular devices. ${ }^{19}$ Example materials include HKUST-1, ${ }^{18,20-22} \mathrm{M}(\mathrm{L})_{2}$ dabco $(\mathrm{M}=\mathrm{Cu}$ or $\mathrm{Zn} ; \mathrm{L}=$ naphthalene dicarboxylate or $\mathrm{F}_{4}$ benzene-dicarboxylate), ${ }^{16}$ and $\mathrm{Fe}$ (pyrazine) $\left[\mathrm{Pt}(\mathrm{CN})_{4}\right] .{ }^{12}$ However, to date, the $\mathrm{LbL}$ approach

Received: July 30, 2013

Published: October 15, 2013 
has not been demonstrated with porphyrin or other lightharvesting or catalytic linkers to form functional films of MOFs. Here, we report the LbL fabrication of films of porphyrin-based MOFs to form films of controlled thickness, orientation, and porosity. We show that the LbL approach provides the first direct route to MOFs containing metal-free porphyrins. Finally, we find that the aligned assemblies of chromophores facilitate long-distance energy transfer to the film periphery as demonstrated by fluorescence measurements.

In addition to the three MOF building blocks (P1, L1, $\mathrm{Zn}_{2}{ }^{4+}$ ), 3-aminopropyl-trimethoxysilane (3-APTMS) was utilized to functionalize the surface of flat silicon and ITO platforms with a presenting layer of amine groups (Figure 1). The hydroxyl-terminated surface of the silicon and ITO samples were derivatized by exposing it to refluxing in a 1:100 (v:v) solution of 3-APTMS in octanol for $20 \mathrm{~min}$. This was followed by rinsing with hexanes and water and then oven drying $\left(70{ }^{\circ} \mathrm{C}, 15 \mathrm{~min}\right)$. Installation of the amine layer was found to be a crucial step, without which the growth of DAMOF was unsuccessful (see Figure S1a). Presumably, the 3APTMS captures the $\mathrm{Zn}^{2+}$, thereby facilitating the growth of subsequent MOF structures. Each cycle of growth on the silicon substrates modified with 3-APTMS consists of successively soaking the substrate in solutions containing $\mathrm{Zn}^{2+}, \mathbf{L 1}$, and finally $\mathbf{P 1}$, all at $40{ }^{\circ} \mathrm{C}$. Before each soaking step, the substrate/film was rinsed with ethanol (EtOH) to remove unreacted precursor ions or molecules and to ensure uniform film growth. Control experiments confirm that all three components are necessary to achieve film growth, a finding that is consistent with assembly of a film containing the components of DA-MOF (Figure S1b). The same procedure was applied to form films of L2-MOF, composed of $\mathrm{Zn}_{2}{ }^{4+}$, 4,4'-bipyridine (P2), and 5,10,15,20-tetrakis(4-carboxyphenyl)porphyrin (L2) (Figure 1).

The number of cycles was controlled using an automated setup adapted from the design of Arslan et al. ${ }^{23}$ As illustrated in Figure 2, this allowed the MOF film thickness to be

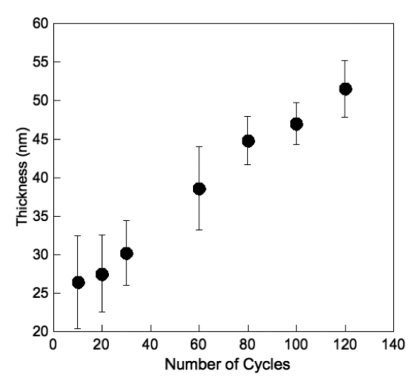

Figure 2. Correlation between DA-MOF film thickness and the number of LbL growth cycles as monitored by profilometry under ambient conditions. The error bars are derived from measurements of film roughness. From 0 to 10 cycles (not shown), the film thickness is on the order of the film roughness.

systematically varied as demonstrated by profilometry measurements. Over the range from 27 to $50 \mathrm{~nm}$, film thickness increases at a rate of $0.24 \mathrm{~nm}$ per cycle, while the roughness decreases from $\sim 6$ to $3 \mathrm{~nm}$ (i.e., from $\sim 2$ to $\sim 1$ structural repeat units films aligned with porphyrin linkers normal to the silicon platform). From 0 to $27 \mathrm{~nm}$, the average film thickness increases more rapidly. Growth, however, appears to emanate from distinct nucleation sites, which may be formed from the cross-linking of the 3-APTMS on silicon to form islands. Thus, films initially display substantial roughness as well as apparently exposed 3-APTMS regions. Eventually the locally nucleated film patches overlap, and the resulting continuous film displays less roughness. Decreases in roughness with increasing cycles of growth in the continuous film region were corroborated via SEM (Figure S3) and AFM measurements (Figure S4). At present, we do not understand the cause of locally nucleated, as opposed to uniform and continuous, initial film growth.

An ensemble of molecular chromophores, once distributed in an ordered structure, can exhibit optical properties that are absent for amorphous ensembles. For example, preferentially oriented chromophores can exhibit excitation-polarizationdependent fluorescence intensities. Conversely, excitationpolarization-sensitive fluorescence can be used to demonstrate alignment. With this in mind, we performed such measurements on LbL-assembled DA-MOF films. Excitation was done at $600 \mathrm{~nm}$, corresponding to the Q-band absorption region of P1. See SI for description of experimental setup. As shown in Figure 3, the examined DA-MOF film exhibits a substantial

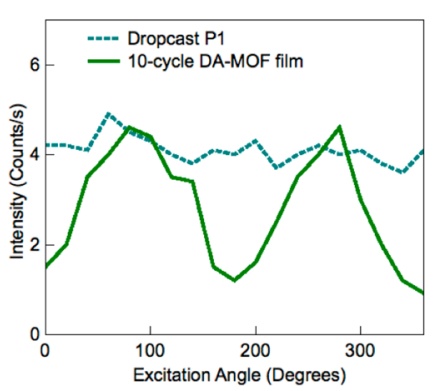

Figure 3. Comparison of fluorescence intensity as a function of the polarization angle of the excitation beam for dropcast P1 on silicon and DA-MOF film on silicon. The excitation wavelength corresponds to the Q-band absorption region of the P1 component.

dependence of the fluorescence intensity on the polarization angle of the excitation beam. The maximum intensity indicates that the overall transition dipole moment averaged from the porphyrin molecules is parallel to the laser polarization, resulting in the highest possibility of excitation. Rotating the polarization by $90^{\circ}$ leads to a decrease of the fluorescence signal, resulting in the lowest possibility of excitation. These results confirm the formation of an aligned porphyrin structure on the silicon surface by LbL.

To assess periodic ordering of the porphyrins, X-ray reflectivity (XRR) scans were measured with a Rigaku ATX$\mathrm{G}$ diffractometer (Figure 4). The scans yielded a weak peak that

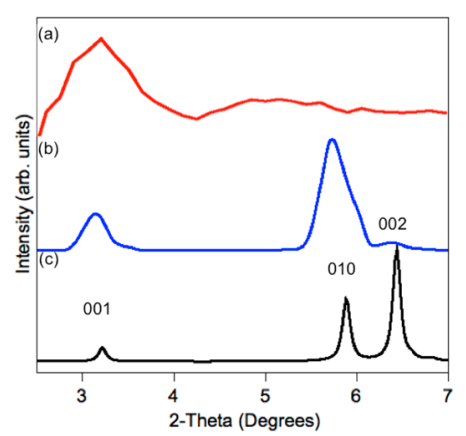

Figure 4. X-ray reflectivity scan of the (a) 50-cycle film in comparison to (b) bulk crystals and (c) a simulated powder pattern of DA-MOF. 
can be indexed as 001 for the 50-cycle film. Since the peak appears at $2 \theta \approx 3.2^{\circ}$, it is consistent with an anticipated interlayer spacing of $\sim 3 \mathrm{~nm}$ and, in turn, consistent with film pillaring by P1. For some applications-in particular, catalysis or photocatalysis-molecular-scale porosity is a highly desirable MOF property.

To quantify the porosity of films, we turned to ellipsometry measurements of evacuated and solvent-filled films. Optical constants of the material were evaluated via ellipsometry measurements. For a porous MOF material, the refractive index, $n$, is a volume-weighted average of the indices for the cavities (vacuum, $n_{\text {vac }}=1$ ) and the framework $\left(n_{\text {fram }}>1\right) .{ }^{22}$ Inserting polarizable molecules into the MOF film cavities will fill the vacuum and increase the overall refractive index. We chose two common MOF solvents as probes: EtOH and $N, N$ dimethylformamide (DMF). From the refractive indices of the filled films, the empty films present solvent-accessible volumes of $\sim 57 \%$ and $85 \%$ for EtOH and DMF, respectively (Table 1 ).

Table 1. Solvent Effects on Estimated Porosity of DA-MOF Film

\begin{tabular}{cccc|}
\hline solvent in DA-MOF & $n($ film $)$ & $n$ (solvent) & estimated porosity \\
\hline none & 1.14 & 1 & $75 \%^{a}$ \\
EtOH & 1.27 & 1.36 & $57 \%$ \\
DMF & 1.42 & 1.43 & $85 \%$
\end{tabular}

${ }^{a}$ Estimated from single-crystal X-ray structure of DA-MOF, and $n$ is the refractive index of the solvent, empty film, or solvent-filled film.

These values are consistent with a value of $\sim 75 \%$ anticipated based on PLATON analysis of the single-crystal X-ray structure of an isolated, synthesis solvent containing sample of DA-MOF.

To begin testing the generality of the LbL approach to assembling porous porphyrin structures, we grew films from $\mathrm{Zn}^{2+}, \mathbf{L 2}$, and P2 to form L2-MOF (Figure 1). ${ }^{24}$ In contrast to the DA-MOF films, L2-MOF films incorporate the nonchromophoric linker (P2) as the pillar and the porphyrin linker (L2) as the organic component of paddlewheel-linked 2D sheets. Polarized fluorescence measurements confirmed that L2 is incorporated and that the resulting ensemble is preferentially oriented (Figure S2). The presence of an XRR peak appears at $2 \theta=6.3^{\circ}$ (Figure S5) indicates an interlayer spacing of $14 \AA$ and is consistent with film pillaring by $\mathbf{P 2}$.

Incorporation of porphyrins into MOFs in free-base form has been problematic, because the porphyrins spontaneously metalate under conventional solvothermal synthesis conditions. Free-base porphyrins are attractive in photochemical applications because they generally display longer singlet excited-state lifetimes than do metalated porphyrins. They also are attractive as starting points for incorporating catalytically active metal ions. While possible indirect routes are offered by solventassisted linker exchange $(\mathrm{SALE})^{24}$ or by syntheses that incorporate metal ions that are subsequently readily removed (e.g., $\mathrm{Zr}^{4+}$ ), ${ }^{27,28}$ no direct route to MOFs containing free-base porphyrins appears to have been described.

We reasoned that the comparatively low temperature employed here for LbL MOF growth $\left(40{ }^{\circ} \mathrm{C}\right)$ would enable nonmetalated porphyrins to be incorporated directly. Visibleregion absorption spectra for the L2-MOF film confirms freebase incorporation as shown by the retention of four porphyrin $\mathrm{Q}$ bands, instead of two $\mathrm{Q}$ bands in the metalated L2 (Figure S6). The Soret band absorption for L2-MOF films is considerably narrower $(\mathrm{fwhm}=20 \mathrm{~nm}$ ) than is the same band for simple dropcast films of $\mathbf{L 2}(\mathrm{fwhm}=86 \mathrm{~nm})$. The difference is indicative of significant aggregation and electronic interaction of porphyrins in the dense dropcast films and effective isolation of chromophoric units in the MOF-based films. ${ }^{29}$

We have previously shown that optical excitation of aligned porphyrins within crystalline MOFs is followed by directional energy migration over appreciable distances (i.e., up to several hundred angstroms). Excitation of a chromophore within free crystals of DA-MOF, for example, is followed by $\sim 2000$ excitonic hops between neighboring, energetically equivalent porphyrin struts. We reasoned that in a thin-film MOF environment, similar excitation could lead to exciton migration (site-to-site hopping) to the periphery of the film. To detect such migration, we installed a supplementary chromophore, a squaraine dye (S1), atop the outermost layer of each investigated film (Figure 5a). It is reasonable to expect the

\section{(a)}

(b)

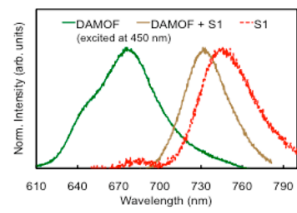

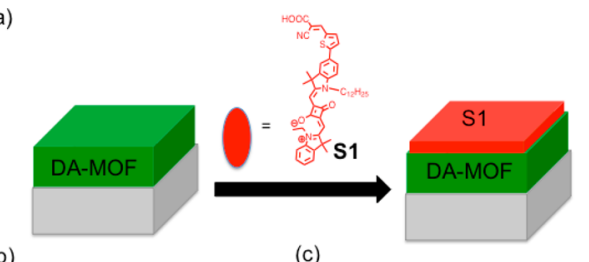

(c)

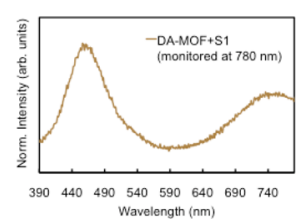

Figure 5. (a) Schematic diagram of preparation of sensitized DAMOF film. (b) Comparison of emission profiles of DA-MOF (green solid), S1 (red solid), and DA-MOF sensitized with S1 (light-green dotted) upon excitation at $450 \mathrm{~nm}$. (c) Excitation profile of the DA$\mathrm{MOF}+\mathrm{S} 1$ film monitored at $780 \mathrm{~nm}$, where the emission from DAMOF is negligible.

carboxylate moiety on $\mathbf{S 1}$ to be capable of binding only to under-coordinated (i.e., tetra-coordinated) Zn nodes present exclusively at the terminus of the MOF film surface. Within the film, these sites are occupied by pillaring pyridyl groups. Importantly, the S1 species is incapable, geometrically, of permeating the crystalline MOF layer, thereby ensuring minimal contact with the interior of the MOF films. Overlap between the emission spectrum of $\mathbf{P 1}$ and the far-red absorption of S1 (Figure S7a) should result in irreversible energy trapping, followed by far-red emission from S1. Additionally, if multistep energy transfer is sufficiently rapid and efficient, trapping of the exciton on S1 will extinguish emission from P1.

Figure $5 \mathrm{~b}$ shows that efficient migration and trapping indeed occur within a 50-cycle film. Figure $5 \mathrm{c}$ is an excitation spectrum (monitored at $\mathrm{S} 1$ emission at $780 \mathrm{~nm}$ ), illustrating that $\mathbf{S 1}$ emission is sensitized by DA-MOF. Peaks at 458 and $750 \mathrm{~nm}$ are indicative of participation of $\mathbf{P 1}$ and S1, respectively, as light-harvesters for $\mathbf{S 1}$ emission. The effect of varying concentrations of $\mathbf{S 1}$ on fluorescence quenching of the DAMOF film was also examined (Figure S8). (Due to aggregation of $\mathbf{S 1}$ at higher concentrations, red-shifting of peaks in the emission spectra was observed.) Porphyrinic sensitization of S1 is also seen with L2-MOF (Figure S9). These findings clearly point to the potential for MOFs to function as true antenna 
structures in molecular materials based devices. They also suggest that MOFs may prove to be versatile testing grounds for complex molecular photonic phenomena.

In summary, we have successfully used an automated LbL assembly technique to obtain luminescent, chromophore aligned, porphyrinic MOF films. The thicknesses of the films can be readily controlled via the number of assembly cycles. Polarized fluorescence excitation measurements confirm that the porphyrin building blocks in thin films of both DA-MOF and L2-MOF are preferentially aligned. XRR scans of LbLassembled films reveal ordering in the direction normal to the film support, with periodicity consistent with pillaring by $\mathbf{P 1}$ or P2. Because the experimental conditions for LbL assembly are much milder than those for conventional synthesis of MOFs, direct incorporation of otherwise readily metalated free-base porphyrins is possible. By infiltrating the pores of DA-MOF with EtOH and DMF, we find that these films are highly porous. Finally, photophysical studies with porphyrinic MOF films that are terminated with a far-red emitting squarine dye show that the films can be used as antennae for light harvesting; efficient energy transfer between porphyrins and ultimately to S1 is evidenced by nearly exclusive emission (fluorescence) from $\mathbf{S 1}$ following selective excitation of P1 units in a 50-cycle film. Taken together, these findings point to the potential utility of porphyrin-based MOF films for light-harvesting and efficient energy transport in solar energy conversion devices.

\section{ASSOCIATED CONTENT}

\section{S Supporting Information}

Experimental procedure and characterization data. This material is available free of charge via the Internet at http://pubs. acs.org.

\section{AUTHOR INFORMATION}

\section{Corresponding Authors}

wiederrecht@anl.gov

o-farha@northwestern.edu

j-hupp@northwestern.edu

Notes

The authors declare no competing financial interest.

\section{ACKNOWLEDGMENTS}

We thank A. Smeigh, S. Kelwaramani, and O. Shekhah for helpful discussions. M.C.S. acknowledges support from the Department of Defense through the National Defense Science \& Engineering Graduate Fellowship (NDSEG) Program. J.T.H. gratefully acknowledges support from Northwestern University and from the U.S. Dept. of Energy, Office of Science, Office of Basic Energy Sciences (grant no. DE-FG02-87ER13808). S.J., who contributed polarized fluorescence measurements, was supported at the Nanoscience \& Technology Division by the ANSER Center, an Energy Frontier Research Center funded by the U.S. Dept. of Energy, Office of Science, Office of Basic Energy Sciences, under award no. DE-SC0001059. Use of Center of Nanoscale Materials is funded by the U.S. Dept. of Energy, Office of Science, Office of Basic Energy Sciences through contract no. DE-AC02- $06 \mathrm{CH} 11357$. We made use of the J.B. Cohen X-Ray Diffraction Facility supported by the MRSEC program of the NSF (DMR-1121262) at the NU Materials Research Center.

\section{REFERENCES}

(1) Lee, C. Y.; Farha, O. K.; Hong, B. J.; Sarjeant, A. A.; Nguyen, S. T.; Hupp, J. T. J. Am. Chem. Soc. 2011, 133, 15858.

(2) Kent, C. A.; Liu, D.; Meyer, T. J.; Lin, W. J. Am. Chem. Soc. 2012, 134, 3991.

(3) Kent, C. A.; Liu, D.; Ma, L.; Papanikolas, J. M.; Meyer, T. J.; Lin, W. J. Am. Chem. Soc. 2011, 133, 12940.

(4) Son, H.-J.; Jin, S.; Patwardhan, S.; Wezenberg, S. J.; Jeong, N. C.; So, M.; Wilmer, C. E.; Sarjeant, A. A.; Schatz, G. C.; Snurr, R. Q.; Farha, O. K.; Wiederrecht, G. P.; Hupp, J. T. J. Am. Chem. Soc. 2012, $135,862$.

(5) Kent, C. A.; Mehl, B. P.; Ma, L.; Papanikolas, J. M.; Meyer, T. J.; Lin, W. J. Am. Chem. Soc. 2010, 132, 12767.

(6) Wang, C.; Lin, W. J. Am. Chem. Soc. 2011, 133, 4232.

(7) Webber, S. E. Chem. Rev. (Washington, DC, U. S.) 1990, 90, 1469.

(8) Sykora, M.; Maxwell, K. A.; DeSimone, J. M.; Meyer, T. J. Proc. Natl. Acad. Sci. U.S.A. 2000, 97, 7687.

(9) Fréchet, J. M. J. J. Polym. Sci., Part A: Polym. Chem. 2003, 41, 3713.

(10) Aratani, N.; Kim, D.; Osuka, A. Acc. Chem. Res. 2009, 42, 1922.

(11) Wang, J.-L.; Wang, C.; Lin, W. ACS Catalysis 2012, 2, 2630.

(12) Otsubo, K.; Haraguchi, T.; Sakata, O.; Fujiwara, A.; Kitagawa, H. J. Am. Chem. Soc. 2012, 134, 9605.

(13) Shekhah, O.; Liu, J.; Fischer, R. A.; Woll, C. Chem. Soc. Rev. 2011, 40, 1081.

(14) Bétard, A.; Fischer, R. A. Chem. Rev. (Washington, DC, U. S.) 2011, 112, 1055

(15) Zacher, D.; Shekhah, O.; Woll, C.; Fischer, R. A. Chem. Soc. Rev. 2009, 38, 1418

(16) Zacher, D.; Yusenko, K.; Bétard, A.; Henke, S.; Molon, M.; Ladnorg, T.; Shekhah, O.; Schüpbach, B.; del os Arcos, T.; Krasnopolski, M.; Meilikhov, M.; Winter, J.; Terfort, A.; Wöll, C.; Fischer, R. A. Chem.-Eur. J. 2011, 17, 1448.

(17) Shekhah, O. Materials 2010, 3, 1302.

(18) Shekhah, O.; Wang, H.; Kowarik, S.; Schreiber, F.; Paulus, M.; Tolan, M.; Sternemann, C.; Evers, F.; Zacher, D.; Fischer, R. A.; Wöll, C. J. Am. Chem. Soc. 2007, 129, 15118.

(19) Araki, K.; Wagner, M. J.; Wrighton, M. S. Langmuir 1996, 12, 5393.

(20) Kreno, L. E.; Hupp, J. T.; Van Duyne, R. P. Anal. Chem. 2010, $82,8042$.

(21) Lu, G.; Farha, O. K.; Kreno, L. E.; Schoenecker, P. M.; Walton, K. S.; Van Duyne, R. P.; Hupp, J. T. Adv. Mater. (Weinheim, Ger.) 2011, 23, 4449 .

(22) Lu, G.; Hupp, J. T. J. Am. Chem. Soc. 2010, 132, 7832.

(23) Arslan, H. K.; Shekhah, O.; Wieland, D. C. F.; Paulus, M.; Sternemann, C.; Schroer, M. A.; Tiemeyer, S.; Tolan, M.; Fischer, R. A.; Wöll, C. J. Am. Chem. Soc. 2011, 133, 8158.

(24) Takaishi, S.; DeMarco, E. J.; Pellin, M. J.; Farha, O. K.; Hupp, J. T. Chem. Sci. 2013, 4, 1509.

(25) Narayan, T. C.; Miyakai, T.; Seki, S.; Dincă, M. J. Am. Chem. Soc. 2012, 134, 12932.

(26) Sun, L.; Miyakai, T.; Seki, S.; Dincă, M. J. Am. Chem. Soc. 2013, 135,8185

(27) Morris, W.; Volosskiy, B.; Demir, S.; Gándara, F.; McGrier, P. L.; Furukawa, H.; Cascio, D.; Stoddart, J. F.; Yaghi, O. M. Inorg. Chem. 2012, 51, 6443

(28) Feng, D.; Gu, Z.-Y.; Li, J.-R.; Jiang, H.-L.; Wei, Z.; Zhou, H.-C. Angew. Chem., Int. Ed. 2012, 51, 10307.

(29) Xu, Z.-X.; Roy, V. A. L.; Liu, Z.-T.; Lee, C. S. Appl. Phys. Lett. 2010, 97, 163301. 